\title{
KM3NeT - ORCA
}

\author{
Jürgen Brunner* \\ Aix Marseille Univ, CNRS/IN2P3, CPPM, Marseille, France \\ E-mail: brunner@cppm.in2p3.fr
}

\section{on behalf of the $\mathrm{KM} 3 \mathrm{NeT}$ collaboration}

ORCA (Oscillations Research with Cosmics in the Abyss) is the low-energy branch of KM3NeT, the next generation underwater Cherenkov neutrino detector in the Mediterranean Sea. Its primary goal is to resolve the long-standing unsolved question of whether the neutrino mass ordering is normal or inverted by measuring matter oscillation effects with atmospheric neutrinos. The ORCA design foresees a dense configuration of KM3NeT detection units, optimised for studying the interactions of neutrinos in seawater at energies between few and $100 \mathrm{GeV}$. Multi-PMT optical modules will exploit the excellent optical properties of deep seawater to accurately reconstruct both cascade (mostly electron neutrinos) and track (mostly muon neutrinos) events. The potential of the ORCA detector both in neutrino mass ordering studies and in obtaining new constraints on other key parameters such as $\theta_{23}$ will be discussed.

XVII International Workshop on Neutrino Telescopes

13-17 March 2017

Venezia, Italy

\footnotetext{
* Speaker.
} 


\section{Introduction}

The main objectives of the KM3NeT Collaboration are the discovery and subsequent observation of high-energy neutrino sources in the Universe [1] and the determination of the mass ordering of neutrinos. To meet this second objective, the KM3NeT Collaboration is building a dense deep sea detector called ORCA (Oscillation Research with Cosmics in the Abyss) as part of a larger network of deep-sea neutrino telescopes in the Mediterranean Sea, in order to study the oscillations of atmospheric neutrinos.

Recent years have seen rapid progress in measuring the parameters governing neutrino oscillations, but several fundamental properties remain to be determined: the octant of $\theta_{23}$ (i.e. whether $\theta_{23}$ is greater or smaller than $45^{\circ}$ ), the value of $\delta_{C P}$ which defines the level of CP-violation in the leptonic sector and the neutrino mass ordering (NMO). A normal ordering (NO) scenario would apply in the case where $m_{1}<m_{2}<m_{3}$, while the ordering $m_{3}<m_{1}<m_{2}$ is referred to as inverted (IO), where the labels $1,2,3$ are defined by the coupling strength to $v_{e}$, i.e. $\left|U_{e 1}\right|^{2}>\left|U_{e 2}\right|^{2}>\left|U_{e 3}\right|^{2}$.

The aim of KM3NeT/ORCA is to perform precision measurements in the energy range 5$30 \mathrm{GeV}$. In this range matter effects are expected to lead to resonance effects in the oscillation pattern of atmospheric neutrinos. These resonances depend on the NMO and occur either in the neutrino or in the anti-neutrino channel. Large volume detectors such as KM3NeT/ORCA cannot distinguish between neutrinos and anti-neutrinos but the asymmetry in the neutrino flux and the cross sections yield an energy and angle dependent difference of the event rates between the two NMO hypotheses. The high statistics and high quality data sample will allow also for other measurements such as the determination of the atmospheric neutrino oscillation parameters $\Delta m_{32}^{2}$ and $\theta_{23}$, the unitarity test of the PMNS mixing matrix by measuring the $v_{\tau}$ appearance rate and to search for exotic signals such as sterile neutrinos or non-standard interactions.

\section{The KM3NeT/ORCA detector}

The ORCA detector will be realised at the French KM3NeT site, off-shore Toulon, at a depth of $2400 \mathrm{~m}$. The complete detector will consist of 115 flexible strings anchored on the seabed and connected to junction boxes that provide power and data communication. Each string comprises 18 digital optical modules (DOMs), which are pressure-resistant glass spheres housing 31 three-inch photomultiplier tubes (PMTs). The high voltage for the PMTs is created insides the DOMs and the PMT signals are digitised there as well. More details on the detector design can be found in [2].

The first main cable, which connects the KM3NeT/ORCA site to the shore has been installed in 2015. One year later the first junction box followed, capable of connecting about 25 detector strings. The first such string has been installed in September 2017.

\section{Simulation}

Neutrino induced interactions have been generated with a software package based on the GENIE [3] neutrino event generator. Electron and muon neutrino events are weighted to reproduce the conventional atmospheric neutrino flux following the Bartol model [4]. All particles emerging from a neutrino interaction vertex are propagated with a GEANT4 based software. Cherenkov light 
is generated from primary and secondary particles. Absorption and scattering in sea water is taken into account when propagating these photons. The generation of "hits" accounts for the DOM and PMT characteristics. The background due to down-going atmospheric muons is generated with the MUPAGE program [5]. In order to reproduce the randomly distributed background PMT hits due to the Cherenkov light from electrons induced by decays of ${ }^{40} K$, single photoelectron hits are added. Also coincidence hits between several PMTs inside the same DOM originating from an individual ${ }^{40} \mathrm{~K}$ decay are taken into account. An uncorrelated hit rate of $10 \mathrm{kHz}$ per PMT and time-correlated noise on each DOM $\left(500 \cdot 10^{2-n} \mathrm{~Hz}\right.$ for $n$-fold coincidences) was added. These are rather conservative values, compared to in situ measurements [6].
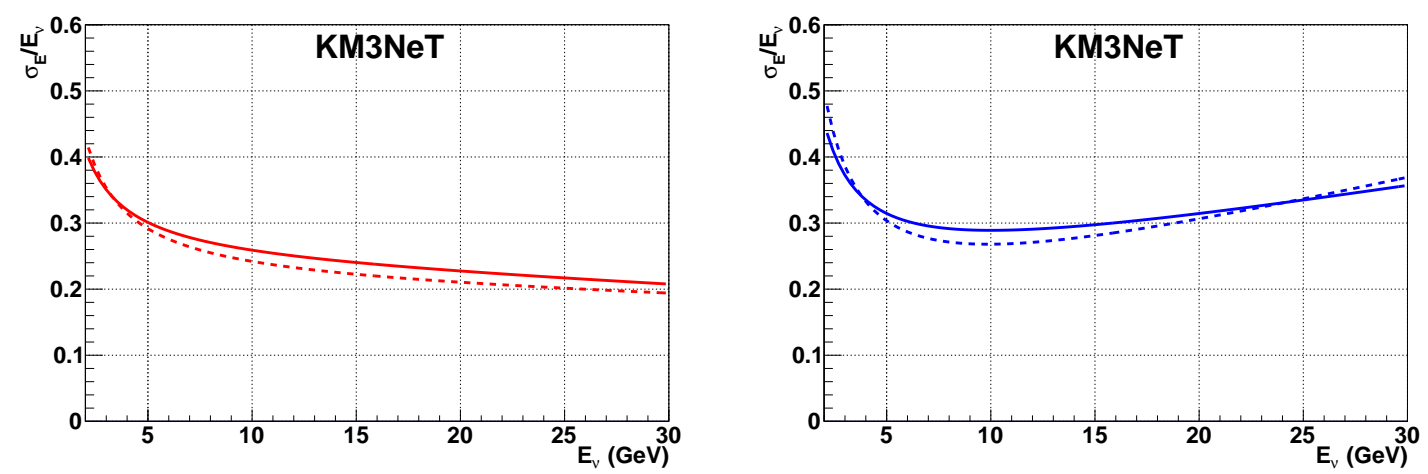

Figure 1: Energy resolution as function of the neutrino energy for $v_{e}-\mathrm{CC}$ (left) and $v_{\mu}$-CC (right); Solid $v$, dashed $\bar{v}$
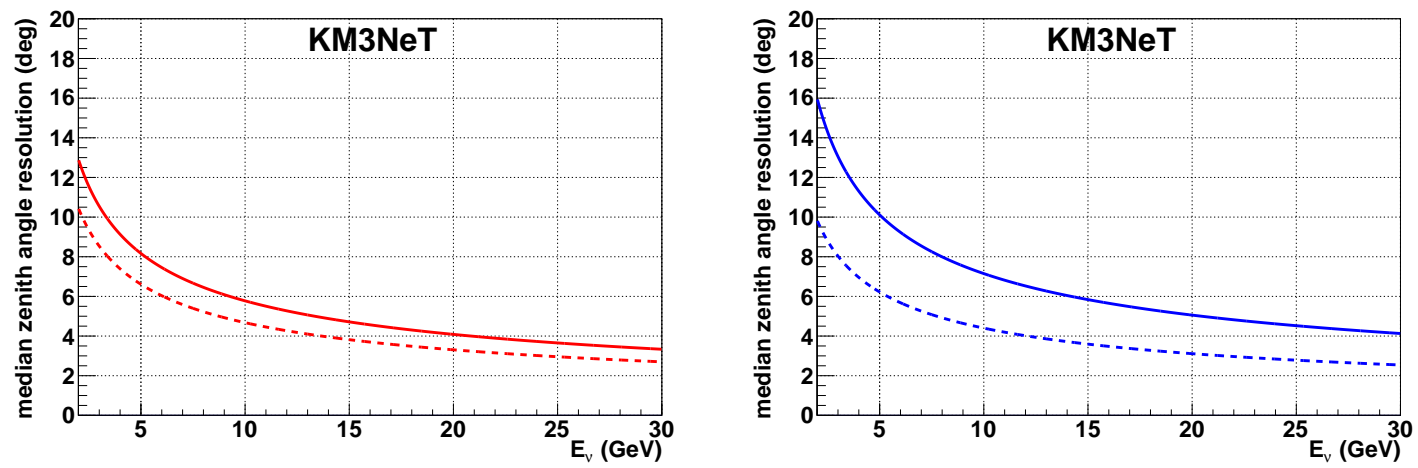

Figure 2: Angular resolution as function of the neutrino energy for $v_{e}-\mathrm{CC}$ (left) and $v_{\mu}$-CC (right) ; Solid $v$, dashed $\bar{v}$

\section{Reconstruction and event selection}

Triggering, event reconstruction and selection are performed for two hypotheses: the "track" signatures assumes the presence of an identifiable muon track, possibly produced in $v_{\mu}$ charged current (CC) interactions or $v_{\tau}$-CC with subsequent muonic decays. Other CC interactions as well as neutral current $(\mathrm{NC})$ reactions produce instead a "shower" signature. The triggering algorithms 
uses clusters of time coincident recorded photons of two PMTs of a single DOM (such coincidence is referred to as $\mathrm{L} 1$ hits). For the shower trigger algorithm, 3 time-correlated L1 hits within a sphere of $43 \mathrm{~m}$, and for the track trigger 4 time-correlated L1s within a tube of $39 \mathrm{~m}$ radius around the track were required. The reconstructions are multi-step algorithms. First the direction of the leading particle and its position in the instrumented volume are approximately determined. The final step is for both signatures a multi-parameter likelihood fit. As a result, the position and direction of the neutrino interaction and an estimate of its energy are obtained. Quality cuts are applied to limit the contamination with misreconstructed atmospheric muons to a level of about $1 \%$ and to ensure a good energy and angular resolution of the final event sample.

\begin{tabular}{||c|r||}
\hline Channel & Events \\
\hline$v_{e}-\mathrm{CC}$ & 17,300 \\
$v_{\mu}-\mathrm{CC}$ & 24,800 \\
$v_{\tau}-\mathrm{CC}$ & 3,100 \\
$\mathrm{NC}$ & 5,300 \\
\hline
\end{tabular}

Table 1: Selected neutrino events per year $(v+\bar{v})$
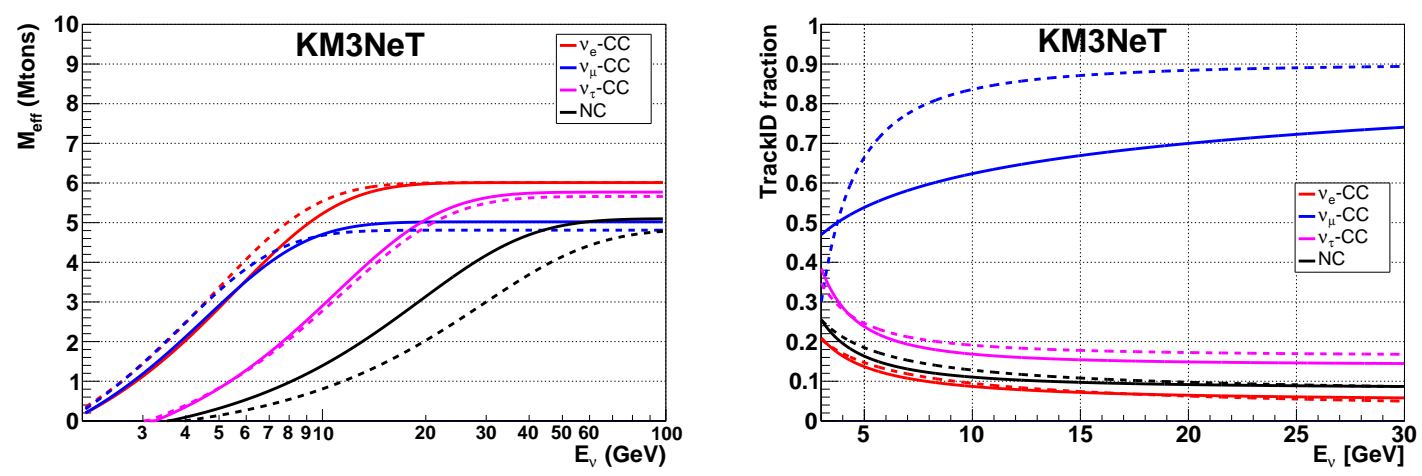

Figure 3: Effective mass (left) and track identification probability (right) as function of the neutrino energy for the three CC interaction channels and for NC; Solid $v$, dashed $\bar{v}$

A summary of the selected events per interaction channel are given in Table 1. The energy resolution of the two main signal channels is shown inf Fig. 1. In the relevant energy range of $5-10 \mathrm{GeV}$ a resolution of better than $30 \%$ is obtained. The degradation of the energy resolution for higher energies in the $v_{\mu}$-CC channel is due to the increasing muon range.

The angular resolution of the final event samples in the same two channels is shown in Fig. 2. It improves roughly with $1 / \sqrt{E_{v}}$ and is for both channels dominated by the angle between neutrino and the outgoing charged lepton.

The efficiency of the detector as function of the neutrino energy is shown in Fig. 3 (left). Here the effective mass is given. Above $10 \mathrm{GeV}$ it reaches the instrumented volume of 6 Mtons for $v_{e}-\mathrm{CC}$ and $v_{\mu}$-CC. The curves are shifted to higher energies for $v_{\tau}$-CC and NC due to the invisible outgoing neutrinos. At $5 \mathrm{GeV}$ about $50 \%$ efficiency is reached.

The last step of the selection procedure is the classification of events into the "shower" and "track" categories. Fig. 3 (right) shows the track identification probability as function of the neu- 
trino energy for all interaction channels. Whereas genuine shower events are classified as such in more than $90 \%$ of the cases above $10 \mathrm{GeV}$, the track identification for $v_{\mu}$-CC events is only at $70 \%$ for $10 \mathrm{GeV}$. The difference between $v_{\mu}$ and $\bar{v}_{\mu}$ is due to their corresponding Bjorken-y distribution, i.e. the different energy transfer from the lepton to the hadron vertex.
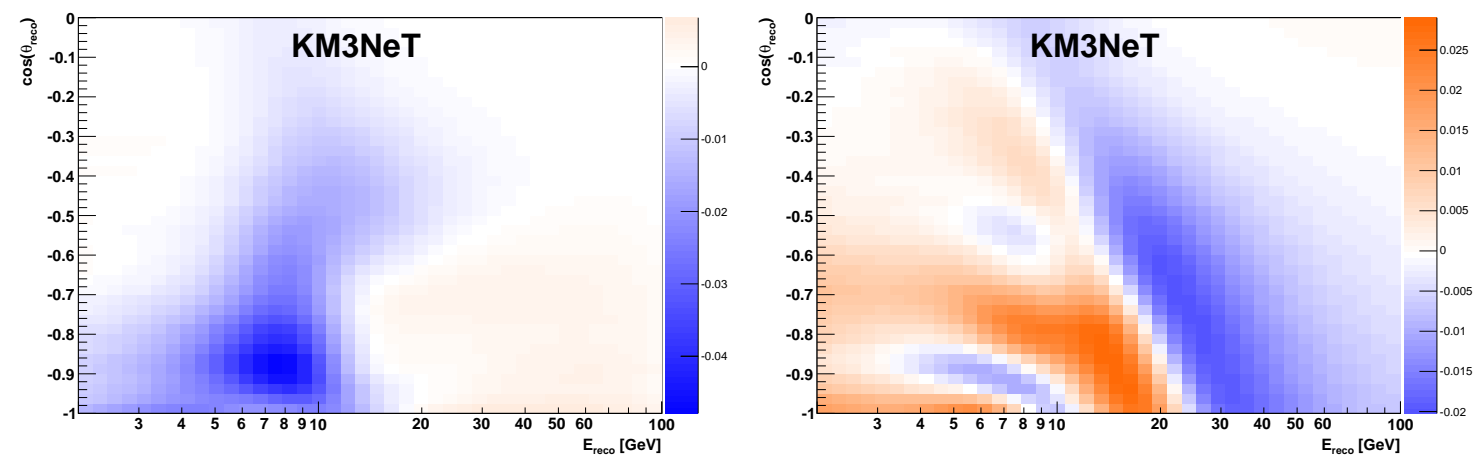

Figure 4: Relative asymmetry of event numbers between the two mass ordering schemes $\left(N_{I O}-N_{N O}\right) / N_{N O}$ as function of the reconstructed energy and zenith angle for the cascade channel (left) and the track channel (right)

\section{Sensitivity to the Neutrino Mass Ordering}

The sensitivity to the Neutrino Mass Ordering is derived from event rates as a function of the reconstructed neutrino energy and zenith angle. By comparing these to the expected rates it will be possible to distinguish between the two mass hierarchy cases. Fig. 4 shows the corresponding event rate asymmetries for both "shower" and "track" signatures including resolutions as well as particle identification imperfections, discussed above. The asymmetries do not exceed few percent per bin but they exhibit a distinct pattern which make it possible to perform a significant measurement of the mass ordering even in the presence of systematic uncertainties.

The sensitivity to the mass hierarchy is calculated using likelihood ratio distributions from pseudo-experiments, while profiling nuisance parameters from interaction rate uncertainties (normalization, $\mathrm{NC}$ scale, energy slope, $v / \bar{v}$ ratio and $v_{\mu} / v_{e}$ ratio) and oscillation parameter uncertainties $\left(\left|\Delta m_{32}^{2}\right|, \theta_{23}, \theta_{13}, \delta_{C P}\right)$. The final figure of merit is the median significance, computed by comparing LLR distributions for true NO and true IO pseudo-experiments. Fig 5 (left) shows the mass hierarchy significance plot as published in [2]. The expected significance depends strongly on the true value of $\theta_{23}$ and to a lesser extent on $\delta_{C P}$. For $\delta_{C} P=0^{\circ}$, the NMO can be measured with more than $3-\sigma$ in three years.

\section{Measurement of oscillation parameters and $v_{\tau}$ normalisation}

Beyond the measurement of the NMO, KM3NeT/ORCA will also be able to measure precisely the dominant parameters responsible for oscillations in the atmospheric scale, $\Delta m_{32}^{2}$ and $\theta_{23}$. A $\chi^{2}$ minimisation is performed while profiling over the same parameters in the NMO analysis. An energy scale shift is implemented as an additional systematic uncertainty. Both $\Delta m_{32}^{2}$ and $\theta_{23}$ 

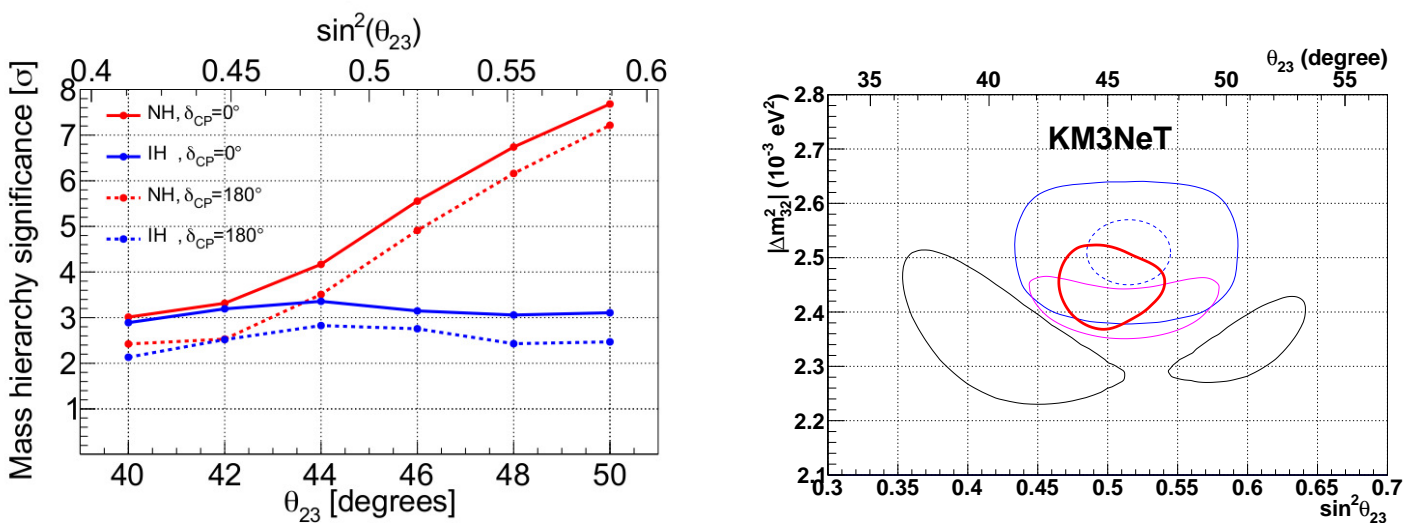

Figure 5: Sensitivity to determine the NMO as function of $\theta_{23}, \delta_{C P}$ and the true NMO (left) and the $1 \sigma$ measurement precision for NO in $\Delta m_{32}^{2}$ and $\theta_{23}$ (right) for ORCA after three years of data taking (red). The measurement contour is compared to current results from Minos [7] (black) and T2K [8] (solid blue) and to predicted measurements of T2K [8] (dashed blue) and Nova [9] (magenta) in 2020. For the latter two and ORCA maximal mixing is assumed for $\theta_{23}$.

are determined under the assumption that the correct NMO has been already identified. The 1- $\sigma$ contour for a test point at $\theta_{23}=45^{\circ}$ obtained after three years of data taking are shown on Fig. 5 (right) in comparison with other existing and planned measurements.

The unitary of the PMNS-matrix can be verified by an independent measurement of the $v_{\tau}$-CC event rate. The contribution of atmospheric $v_{\tau}$ for $E_{v}<100 \mathrm{GeV}$ is negligibly small. Therefore $v_{\tau}$ events can only be created via neutrino oscillations from other flavours. The experimental signature of $v_{\tau}$ appearance is an additional contribution of "shower" events with energies of 10$30 \mathrm{GeV}$ depending on zenith angle. A quantitative measurement can be performed by allowing the normalisation of the $v_{\tau}$ rate to vary in the fit. Fig. 6 shows the 1,2,3 and 5- $\sigma$ measurement contours for such a $v_{\tau}$ normalisation as function of measurement time where a normalisation of one corresponds to the standard 3-flavour scheme. Within one year the $v_{\tau}$ rate can be determined to better than $10 \%$. The presence of additional sterile neutrino flavours would yield a lower value of the $v_{\tau}$ normalisation, if the new mass eigenstates couple sufficiently strong to the $v_{\tau}$.

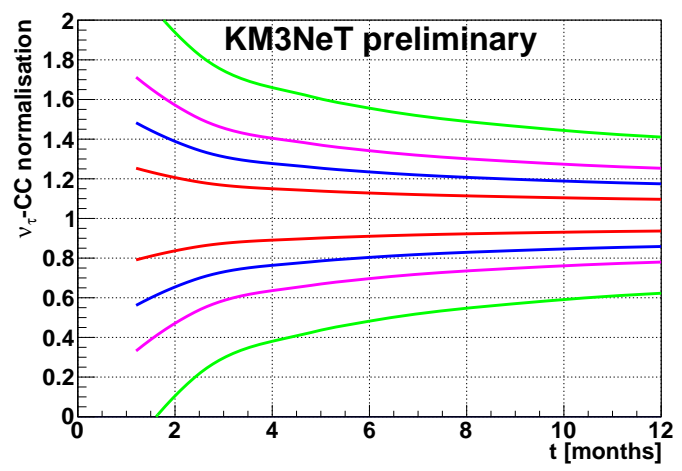

Figure 6: Precision of measuring $v_{\tau}$ appearance and to constrain the normalisation of the $v_{\tau}$ flux within the first year of data taking. The color lines indicate the 1,2,3 and 5- $\sigma$ contours. 


\section{Coincidence rates and SuperNovae neutrinos}

The presence of 31 individual PMTs in each DOM allows to count photons on nsec timescales and to distinguish different Cherenkov light sources simply by exploiting the detected photon (i.e. hit) multiplicity on an individual DOM without invoking any multi-DOM reconstruction and analysis algorithms. Individual uncorrelated hits originate from far away (up to a distance of the water absorption length) radioactive decays or bioluminescence. Hit multiplicities of 2-6 are created by ${ }^{40} \mathrm{~K}$ decays in the vicinity of the DOM. The observed coincidence rates agree well with simulations of such decays as seen on Fig. 7 (left). For hit multiplicities of 7 and higher, rates are dominated by Cherenkov light from down-going atmospheric muons. A clear depth dependence of the muon rate can be seen by comparing the coincidence levels of two DOMs at a depth of 3400m (S1F1) and $2800 \mathrm{~m}(\mathrm{~S} 2 \mathrm{~F} 18)$.
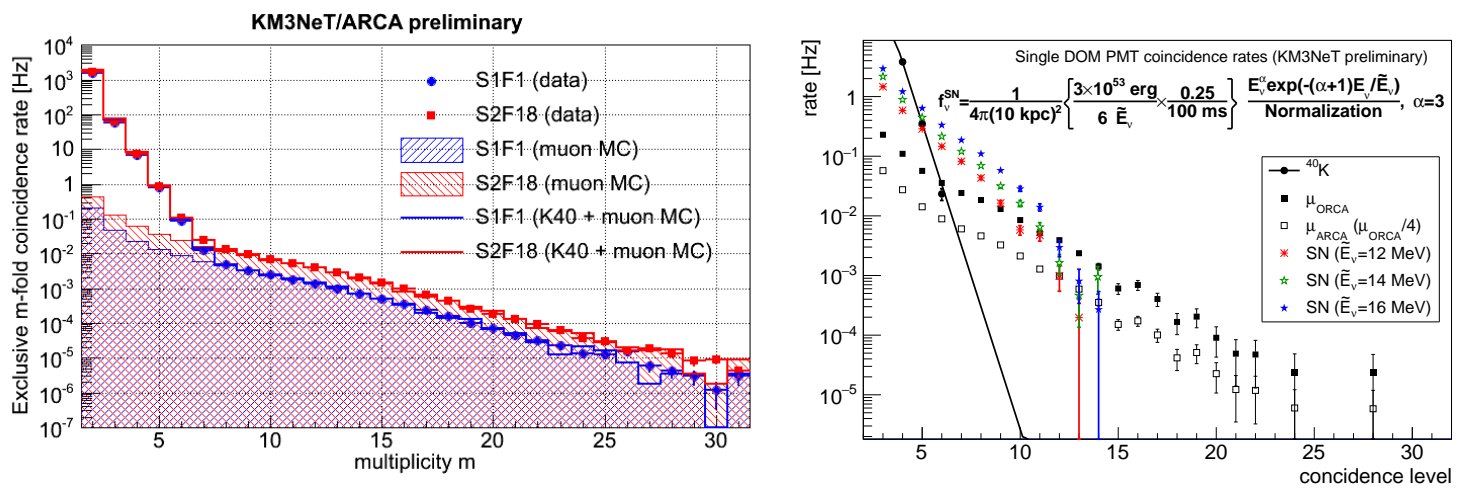

Figure 7: Hit multiplicities measured on two selected DOMs compared to simulations from ${ }^{40} K$ decays and atmospheric muons (left) with an additional signal from SuperNovae neutrinos (right). See text for details.

Signals from a Galactic SuperNova collapse could also be identified via the hit multiplicities of individual DOMs. On Fig. 7 (right) simulations from ${ }^{40} K$ decays and atmospheric muons at the KM3NeT/ARCA and KM3NeT/ORCA site are confronted with an expected neutrino signal of a SuperNova at a distance $10 \mathrm{kpc}$ for different assumptions on the neutrino spectrum. At hit multiplicities between 5-10 the additional contribution from the SuperNova neutrino is clearly identifiable.

\section{Conclusions}

The KM3NeT project is on the French roadmap of Research Infrastructures and was recently selected for the 2016 roadmap of the European Strategy Forum on Research Infrastructures (ESFRI). It is also strongly supported by the APPEC roadmap. The first KM3NeT/ORCA string was deployed in September 2017 and is taking data since. Funding permitting, the rest of the detector could be completed by mid-2020. The projected sensitivities guarantee a 3- $\sigma$ median significance determination of the NMO in 3 years, in addition to competitive measurements of other oscillations parameters. 


\section{References}

[1] M. Taiuti on behalf of the KM3NeT Collaboration, this issue.

[2] S. Adrián-Martínez et al. [KM3NeT Collaboration], J. Phys. G, 43 (8) (2016) 084001.

[3] C. Distefano, EPJ Web Conf. 116 (2016) 08001.

[4] G. D. Barr et al., Phys. Rev. D70 (2004) 023006.

[5] Y. Becherini et al., Astropart. Phys. 25 (2006) 1.

[6] S. Adrián-Martínez et al. [KM3NeT Collaboration], Eur. Phys. J. C74 (2014) 3056.

[7] P. Adamson et al. [MINOS Collaboration], Phys. Rev. Lett. 112 (2014) 191801.

[8] K. Abe et al. [T2K Collaboration], Phys. Rev. D 91 (2015) 7, 072010.

[9] [NOVA Collaboration], http://www-nova.fnal.gov/plots_and_figures/ 1600_Theta23_DeltaMsqr23/100-contours.png 